Article

\title{
Optimization and Model Validation of Operation Control Strategies for a Novel Dual-Motor Coupling-Propulsion Pure Electric Vehicle
}

\author{
Jianjun Hu ${ }^{1,2, *}$, Lingling Zheng ${ }^{2}$, Meixia Jia ${ }^{2}$, Yi Zhang ${ }^{3}$ and Tao Pang ${ }^{2}$ \\ 1 State Key Laboratory of Mechanical Transmission, Chongqing University, Chongqing 400044, China \\ 2 College of Automotive Engineering, Chongqing University, Chongqing 400044, China; \\ lingling66zh@gmail.com (L.Z.); jiameixia23@163.com (M.J.); 18323216353@163.com (T.P.) \\ 3 College of Engineering and Computer Science, University of Michigan-Dearborn, Dearborn, MI 48128, USA; \\ anding@umich.edu \\ * Correspondence: hujianjun@cqu.edu.cn; Tel.: +86-139-9607-3282
}

Received: 17 February 2018; Accepted: 22 March 2018; Published: 27 March 2018

\begin{abstract}
The strict operational condition of driving motors for vehicles propels the development of more complicated configurations in pure electric vehicles (PEVs). Multi-power-source powertrain configurations are one of the efficient technologies to reduce the manufacturing difficulty of driving motors. However, most of the existing studies are predominantly focused on optimal designs of powertrains and power distribution between the engine and motor of hybrid electric vehicles, which are not appropriate for PEVs. This paper proposes a novel dual-motor coupling-propulsion powertrain system that improves the dynamic and economic performance of the powertrain system in PEVs. The proposed powertrain system can realize both the single-motor driving mode and dual-motor coupling driving mode. The driving modes are divided and a power distribution strategy for the different driving modes based on an optimal system efficiency rule is employed, which enhances the performance of the proposed system. Further, a mode-switching strategy that ensures driving comfort by preventing jerk during mode switching is incorporated into the system. The results of comparative evaluations that were conducted using a dual-motor electric vehicle model implemented in MATLAB/Simulink, indicate that the mileage and dynamic performance of the proposed powertrain system are significantly better than those of the traditional single-motor powertrain system.
\end{abstract}

Keywords: dual-motor pure electric vehicle; coupling-propulsion powertrain; mode division; power distribution; mode switch

\section{Introduction}

The environmental degradation and greenhouse effect that is caused by the burning of non-renewable fossil fuels are detrimental to both society and sustainable development. Nevertheless, the transportation system currently depends substantially on fossil fuels [1]. Low-emission pure electric vehicles are among the most effective technologies to alleviate such dependency and promote further developments of green transportation [2]. The design of the powertrain structure and the drive control strategy of pure electric vehicles (PEVs) are important for high economic, dynamic, and ride comfort performances [3].

There are two types of powertrains for PEVs: single-power-source powertrain and multi-power-source powertrain. The former type is simple but it relies heavily on high-performance motors, which interferes with the balance between the dynamics and the economy of electric vehicles [4]. The latter type can be further classified into motor distributed powertrain system and motor coupling powertrain system. Motor 
coupling powertrain systems use multiple motors, instead of one motor, to implement multiple driving modes, and utilize coupling mechanisms to carry out rational composition and decomposition for motor power. Suitable driving modes can be selected to match different driving conditions. This enables PEVs to operate efficiently for extended periods with better energy-saving potential than ordinary vehicles, while satisfying the requirement for dynamic vehicle performance [5].

Because of its multi-mode nature, which includes mode division strategy, power distribution strategy, and mode-switch control strategies, the drive control strategy for multi-motor coupling in pure electric vehicles is more complicated than that of electric vehicles with a single power source. The mode division strategy for PEVs defines the mode boundary that is based on dynamic requirements and economic performance of vehicles. The efficiency of system components, such as the driving motors, are often applied to optimize the mode boundary [6], which can improve the economic performance of vehicles. However, the efficiency of other system components was not considered. On the other hand, methods such as instantaneous optimization $[7,8]$ and global optimization $[6,9]$ can also be applied to optimize the trade-off between the dynamics and efficiency [10]. The two methods can dramatically improve the performance of vehicles based on the cost function, but the high-computational cost and high dependence on driving cycles impede the practical application of the instantaneous optimization and global optimization [11,12]. Therefore, in order to consider the characteristic of interaction of interaction between the system components and avoid the dependence on driving cycles, this paper utilizes the optimal system efficiency method to divide the mode boundary.

Power distribution strategies are employed to control the power of power sources after confirming the driving mode. Minimum vehicle emission rules and minimum energy-loss rules are usually applied in these strategies [13-17]. Power distribution control for multi-power-sources systems on hybrid electric vehicles focuses on balancing the power between engine and motors [18,19]. As for PEVs, the main issue is to ascertain the power of each motor under certain vehicle operation condition [20].

Mode-switch control strategies coordinate the control of multiple power sources during mode switching, such that jerk during switching is reduced and modes can be switched smoothly [21,22]. Current methods include motor torque compensation [23], neural network optimization [24], and optimal trajectory [25].

Existing research is predominantly focused on optimal designs of powertrains and power distribution between the engine and motor of hybrid electric vehicles. However, such research is not applicable to the development of the proposed multi-power-source powertrain that is required for PEVs owing to their unique structures and power source requirements. In this paper, a novel dual-motor coupling-propulsion powertrain system (NDMPS) is proposed for PEVs. This system realizes four driving modes: single motor 1 drive (M1), single motor 2 drive (M2), two motors torque coupling drive (M3), and two motors speed coupling drive (M4). To confirm the driving modes in different driving conditions, their working ranges were identified and analysed. Further, a mode division strategy based on optimal system efficiency was implemented. In addition, a power distribution strategy that realizes power distribution during the two motor driving modes was also developed based on an optimal system efficiency rule. The switch processes between the driving modes have been analysed and enhanced in order to improve the driving comfort of PEVs. A simulated PEV model equipped with the proposed drive system verified its dynamic, economic, and riding comfort performances.

\section{Proposed Novel Dual-Motor Coupling-Propulsion Powertrain System (NDMPS)}

The structure of the proposed NDMPS is shown in Figure 1. This system comprises a main motor (MG1), auxiliary motor (MG2), brake (B1), clutch (C1, C2, C3), planetary gear system, and gear transmission. MG1 is connected to the ring gear of the planetary gear system by $\mathrm{C} 1$ and connects with the output shaft of the system by gear 1 and C2. MG2 is connected to the sun gear, while the planet carrier is linked to the output shaft through gear 2 and C3. 


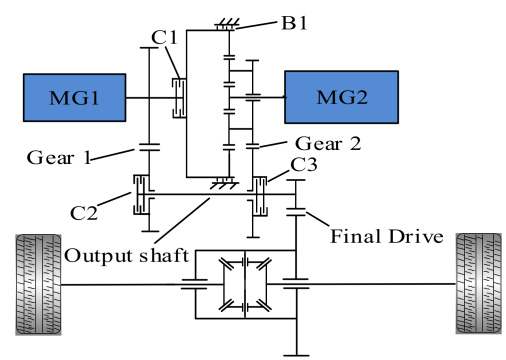

Figure 1. The structure of novel dual-motor coupling-propulsion powertrain system (NDMPS).

By controlling the operation of the motor, brake, and clutch, this system achieves four driving modes. The operational status of components in each driving mode is presented in Table 1.

The basic parameters and performance indicators of a PEV equipped with NDMPS are presented in Table 2 (based on the National Standard GB/T 28382-2012).

Table 1. Operational status of components in the different driving modes.

\begin{tabular}{cclllll}
\hline Mode & MG1 & MG2 & C1 & C2 & C3 & B1 \\
\hline M1 & DRIVE & IDLE & OFF & ON & OFF & OFF \\
M2 & IDLE & DRIVE & OFF & OFF & ON & ON \\
M3 & DRIVE & DRIVE & OFF & ON & ON & ON \\
M4 & DRIVE & DRIVE & ON & OFF & ON & OFF \\
\hline
\end{tabular}

Table 2. Basic parameters and performance indicators of a pure electric vehicles (PEV) equipped with NDMPS.

\begin{tabular}{ccc}
\hline Item & Description & Value \\
\hline \multirow{4}{*}{ Basic parameters } & Fully loaded mass, $\mathrm{m}(\mathrm{kg})$ & 1700 \\
& Windward area, $\mathrm{A}\left(\mathrm{m}^{2}\right)$ & 2.16 \\
& Drag coefficient & 0.29 \\
& Tire rolling radius, $\mathrm{r}(\mathrm{m})$ & 0.345 \\
& Rolling resistance coefficient, $\mathrm{f}$ & 0.015 \\
& Rotational mass coefficient, $\delta$ & 1.04 \\
\hline \multirow{3}{*}{ Performance indicators } & Maximum speed $(\mathrm{km} / \mathrm{h})$ & 150 \\
& Acceleration time of $0 \sim 50 \mathrm{~km} / \mathrm{h}(\mathrm{s})$ & 5 \\
& Acceleration time of $0 \sim 100 \mathrm{~km} / \mathrm{h}(\mathrm{s})$ & 15 \\
& Maximum climbing degree of $15 \mathrm{~km} / \mathrm{h}(\%)$ & 30 \\
& Mileage of $60 \mathrm{~km} / \mathrm{h}$ cruising $(\mathrm{km})$ & 150 \\
\hline
\end{tabular}

The parameters of the powertrain system and the traction battery are matched based on the performance indicators in Table 2 and the results are listed in Table 3.

Table 3. Parameters of the powertrain system.

\begin{tabular}{ccc}
\hline Component & Parameter & Value \\
\hline \multirow{2}{*}{ MG1 } & Rated/peak power $(\mathrm{kW})$ & $22 / 46.5$ \\
& Rated/maximum speed $(\mathrm{rpm})$ & $2880 / 5636$ \\
& Rated/maximum torque $(\mathrm{N} \cdot \mathrm{m})$ & $73 / 154$ \\
\hline \multirow{2}{*}{ MG2 } & Rated/peak power $(\mathrm{kW})$ & $10 / 20.5$ \\
& Rated/maximum speed $(\mathrm{rpm})$ & $3300 / 8338$ \\
& Rated/maximum torque $(\mathrm{N} \cdot \mathrm{m})$ & $29 / 59$ \\
\hline Planetary gear & Ratio between ring gear and sun gear, $\mathrm{k}$ & 2.6 \\
\hline Gear 1 & Ratio $\mathrm{i}_{1}$ & 2.92 \\
\hline Gear 2 & Ratio i & 1.2 \\
\hline Final drive & Ratio $\mathrm{i}_{0}$ & 4.05 \\
\hline \multirow{2}{*}{ Battery pack } & Type & Li-ion \\
& Rated voltage $(\mathrm{V})$ & 333 \\
\hline
\end{tabular}




\section{Driving Mode Division and Power Distribution}

The economic performance of a vehicle is directly related to its operational efficiency. Therefore, it is important to divide the work areas of each driving mode on the basis of the optimal system efficiency rule.

\subsection{Efficiency and Dynamics Model for the Powertrain System}

The efficiency of the powertrain system $\eta_{\text {sys }}$ is modelled as follows:

$$
\eta_{\text {sys }}=P_{\text {out }} / P_{\text {in }}=\frac{F_{r} \cdot u / 1000}{\left(T_{m 1} \cdot \omega_{m 1} / \eta_{m 1}+T_{m 2} \cdot \omega_{m 2} / \eta_{m 2}\right) /\left(\eta_{p} \cdot \eta_{\text {gear }} \cdot \eta_{\text {bat }} \cdot \eta_{\text {inv }}\right)},
$$

where $P_{\text {out }}$ and $P_{\text {in }}$ are the output power and input power of the powertrain system, respectively. The term $F_{r}$ represents all the vehicle loads, including the rolling resistance force, the aerodynamic drag force, the gravity force on graded road, and accelerating resistance force. $u$ represents the vehicle speed. $T_{m 1}$ and $T_{m 2}$ are the torque of MG1 and MG2, respectively. $\omega_{m 1}$ and $\omega_{m 2}$ are the rotational speed of MG1 and MG2, respectively. $\eta_{m 1}$ and $\eta_{m 2}$ are the efficiency of MG1 and MG2. $\eta_{\text {bat }}, \eta_{\text {inv }}, \eta_{\text {gear }}$, $\eta_{p}$ are the efficiency of the battery pack, inverter, transmission gear, and planetary gear, respectively.

In addition to the driving conditions of the PEV, the efficiency of the powertrain system is also related to the efficiency of the driving motors, charge-discharge efficiency of the battery, conversion efficiency of inverters, the transmission efficiency of planetary gear, and the output power of the motors. The efficiency models of the motors and battery are obtained from experimental data. These data are processed to be look up tables for model simulation. Therefore, it is necessary to investigate the output power of the motors in each driving mode.

\subsubsection{System Dynamics in M1 and M2}

The system dynamics of M1 and M2 can be modelled as Equations (2) and (3), respectively. The output power is determined by the requirement of the wheel rotational speed $\omega_{r}$ and the required wheel torque $T_{r}$ :

$$
\begin{gathered}
\left\{\begin{array}{l}
\left(\frac{I_{r}}{i_{0}{ }^{2} \cdot i_{1}{ }^{2}}+I_{m 1}\right) \dot{\omega}_{m 1}=T_{m 1}-\frac{T_{r}}{i_{0} \cdot i_{1} \cdot \eta_{0} \cdot \eta_{1}}, \\
\omega_{m 1}=i_{0} \cdot i_{1} \cdot \omega_{r}
\end{array}\right. \\
\left\{\begin{array}{l}
\left(\frac{I_{r}}{(k+1)^{2} \cdot i_{0}{ }^{2} \cdot i_{2}{ }^{2}}+I_{m 2}\right) \dot{\omega}_{m 2}=T_{m 2}-\frac{T_{r}}{(k+1) \cdot i_{2} \cdot i_{0} \cdot \eta_{p} \cdot \eta_{2} \cdot \eta_{0}}, \\
\omega_{m 2}=(k+1) \cdot i_{2} \cdot i_{0} \cdot \omega_{r}
\end{array}\right.
\end{gathered}
$$

where $I_{r}, I_{m 1}$, and $I_{m 2}$ are the moment of inertia of the wheels, MG1, and MG2, respectively. $\eta_{0}, \eta_{1}$, and $\eta_{2}$ are the transmission efficiency of the final gear, gear1, and gear2, respectively. The meanings of $i_{0}, i_{1}, i_{2}, k$ are reported in Table 3 .

\subsubsection{System Dynamics in M3}

How to distribute the required torque $T_{r}$ to two motors is a key question in calculating the motor working points and the optimal efficiency of the powertrain system in M3. The system dynamics are modelled as follows:

$$
\left\{\begin{array}{l}
\left(\frac{I_{r}}{i_{0}{ }^{2}}+I_{m 2}(k+1)^{2} \cdot i_{2}{ }^{2}+I_{m 1} \cdot i_{1}{ }^{2}\right) \dot{\omega}_{0}=T_{m 2} \cdot(k+1) \cdot i_{2} \cdot \eta_{p} \cdot \eta_{2}+T_{m 1} \cdot i_{1} \cdot \eta_{1}-\frac{T_{r}}{i_{0} \cdot \eta_{0}} \\
\omega_{0}=i_{0} \cdot \omega_{r}=\frac{\omega_{m 2}}{(k+1) \cdot i_{2}}=\frac{\omega_{m 1}}{i_{1}}
\end{array},\right.
$$

In Equation (4), $\omega_{0}$ is the rotational speed of the final gear. The rotational speeds of the motors, $\omega_{\mathrm{m} 1}$ and $\omega_{m 2}$, are proportional to the required rotational speed $\omega_{r}$. The output torques of the motors, $T_{m 1}$ and $T_{m 2}$, are coupled with the required wheel torque $T_{r}$, which can be regulated within certain 
ranges. At this point, the rotational speed of MG1 and MG2 can be calculated from the vehicle velocity, and the torque of MG1 and MG2 for the optimal system efficiency can be determined by an ergodic process [26], as shown in Figure 2 where the initial value of the current maximum system efficiency is assumed to be zero. The minimum and maximum output torque of these motors can be determined from the external characteristic curve of the motors.

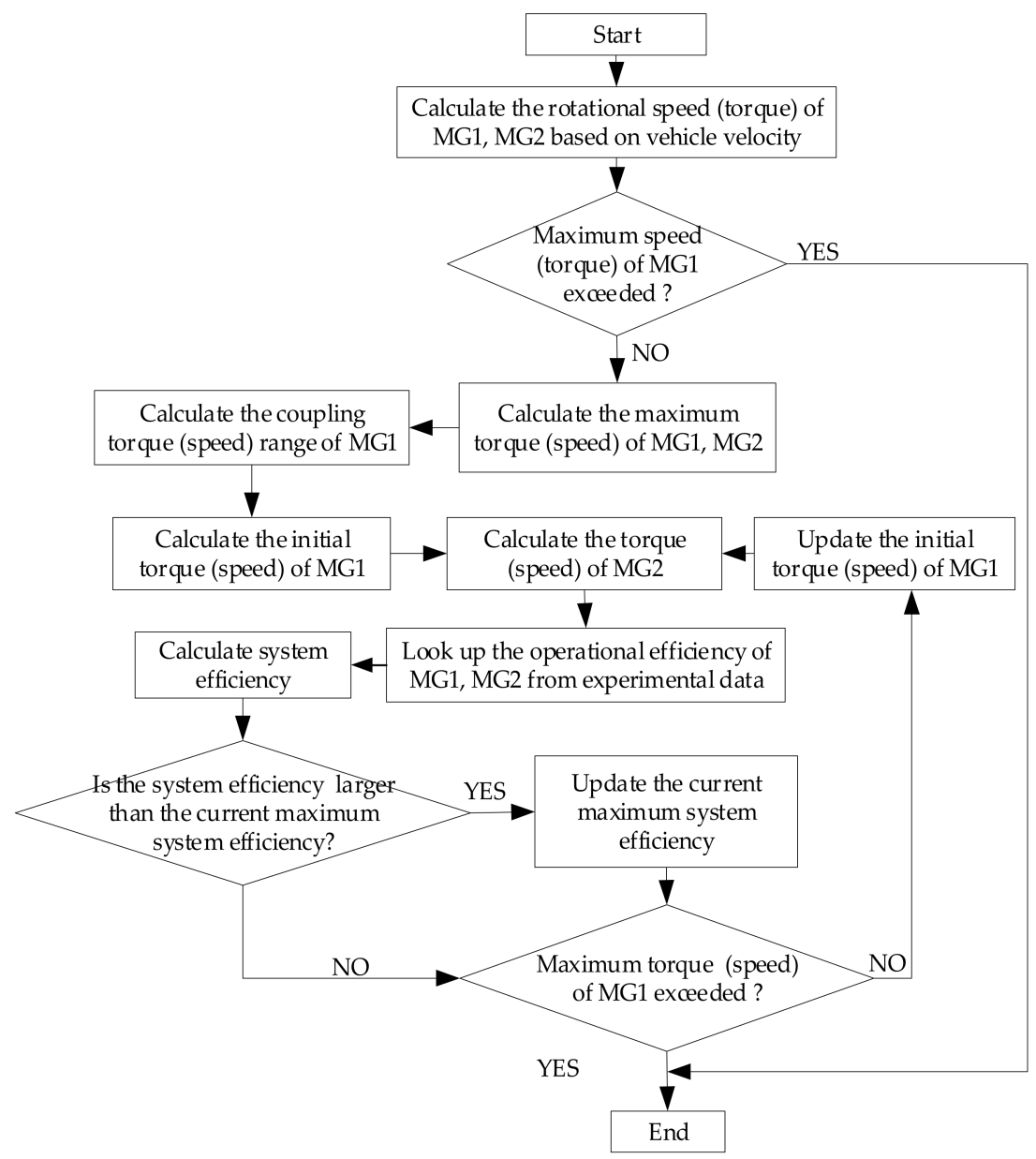

Figure 2. Flowchart for power distribution in two motors torque coupling drive (M3) (two motors speed coupling drive (M4)).

\subsubsection{System Dynamics in M4}

The system dynamics in M4 are modelled as Equation (5). In the speed coupling mode, the output torques of the motors $T_{m 1}$ and $T_{m 2}$ are proportional to the required vehicle torque $T_{r}$. The rotational speeds of the motors $\omega_{\mathrm{m} 1}$ and $\omega_{\mathrm{m} 2}$ are coupled with the required rotational speed $\omega_{r}$, which can be regulated within certain ranges. The characteristic of the powertrain system in M4 is similar to that in M3. Thus, the power distribution calculation process for the motors in M3 can be applied to those in M4 (Figure 2).

$$
\left\{\begin{array}{l}
I_{r} \dot{\omega}_{r}+I_{m 1} \cdot \dot{\omega}_{m 1} \cdot(k+1) \cdot i_{0} \cdot i_{2} / k=T_{m 1} \cdot(k+1) \cdot i_{0} \cdot i_{2} \cdot \eta_{2} \cdot \eta_{0} / k-T_{r} \\
I_{r} \dot{\omega}_{r}+I_{m 2} \cdot \dot{\omega}_{m 2} \cdot(k+1) \cdot i_{0} \cdot i_{2}=T_{m 2} \cdot(k+1) \cdot i_{0} \cdot i_{2} \cdot \eta_{2} \cdot \eta_{0}-T_{r} \\
\omega_{m 1} \cdot k+\omega_{m 2}=i_{0} \cdot i_{2}(k+1) \cdot \omega_{r}
\end{array},\right.
$$




\subsection{Analysis of Driving Mode Division}

Following the establishment of the motor power model in different driving modes, the system efficiency maps and mode distribution results, can be calculated and then illustrated in Figure 3. Figure 3a shows the system efficiency maps for different driving modes under various driving conditions. These system efficiency maps are projected onto the speed-acceleration coordinate plane. For a given point on the vehicle speed-acceleration plane, the driving mode with the maximum system efficiency is selected as the optimal mode. The maximum efficiency $\eta_{\text {opt }}$ is calculated using Equations (6) and (7). Then, the operating boundary of the four modes of NDMPS is obtained, as shown in Figure 3b.

$$
\begin{aligned}
& \eta_{o p t}=\max \left(\eta_{s y s_{-} M 1}, \eta_{\text {sys_M2 }}, \eta_{\text {sys_M3 }}, \eta_{\text {sys_M4 }}\right), \\
& \left\{\begin{array}{l}
0 \leq \omega m 1 \leq \omega m 1 \_\max \\
0 \leq \omega m 2 \leq \omega m 2 \text { max } \\
0 \leq T m 1(\omega m 1) \leq T \max \_1(\omega m 1) \\
0 \leq T m 2(\omega m 2) \leq T \max \_2(\omega m 2) \\
\text { SOCmin } \leq \text { SOC } \\
0 \leq \mid \text { Pbat } \mid \leq \text { Pbat_max }
\end{array}\right.
\end{aligned}
$$

where $\eta_{\text {sys_M1 }}, \eta_{\text {sys_M2 }}, \eta_{\text {sys_M3 }}, \eta_{\text {sys_M4 }}$, are the system efficiency of M1, M2, M3, and M4, respectively. $\omega_{m 1 \_m a x}$ and $\omega_{m 2 \_}$max are the maximum rotational speed of MG1 and MG2 respectively. SOC represents the state of charge of battery pack and $S O C_{\min }$ is the minimum value of discharge depth. $P_{b a t}$ is the battery power and $P_{b a t \_m a x}$ is the peak battery power.

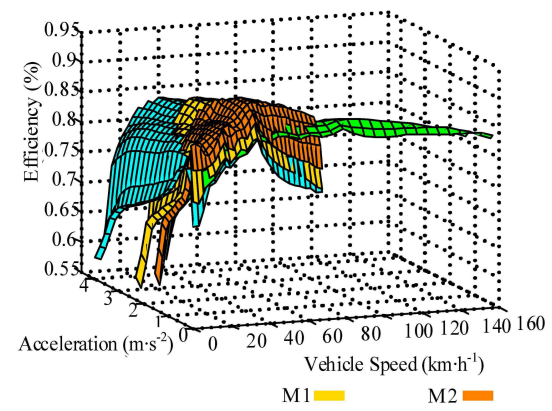

(a)

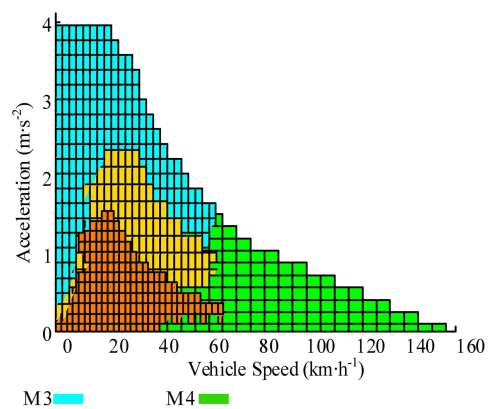

(b)

Figure 3. (a) System efficiency maps of the four modes; and, (b) Operating boundaries of the four modes.

To improve the operating efficiency of the system controller, the results of the mode division is pre-calculated. Figure 4 shows the simplified mode boundary curve. The result is stored in the controller and used as the threshold value of the mode switch.

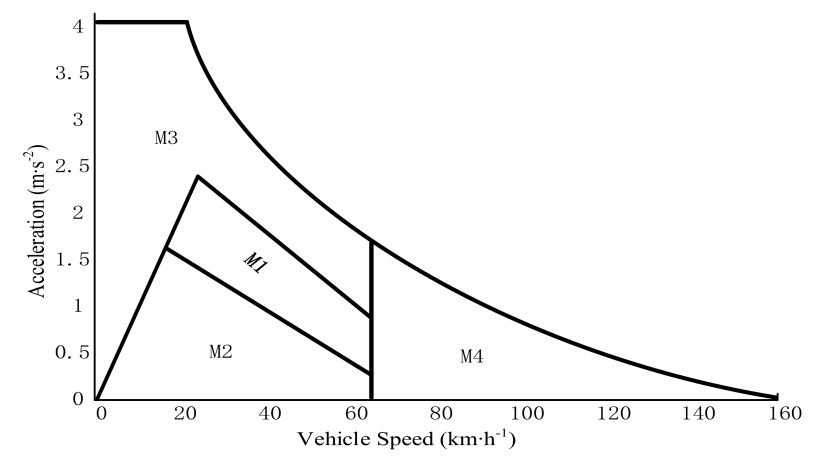

Figure 4. Simplified mode boundary curve. 
Based on the above-mentioned mode division logic, a reasonable working mode with optimal efficiency is selected in terms of the velocity and the acceleration of the vehicle; the acceleration is calculated using the required torque of the vehicle.

\subsection{Power Distribution for Motors}

In the single-motor driving modes, the motor power is directly determined by the required speed and torque of the vehicle. In dual-motor torque coupling driving mode and speed coupling driving mode, the motor power can be regulated within certain ranges. Figure 5 shows the reference torque and the speed of two motors under a given vehicle speed and acceleration, where the driving mode is obtained by referring to Figure 4. The motor torque in M3 and the motor speed in M4 with maximum system efficiency can be calculated by referring to Figure 2 . The energy recovery performance of the motors is also considered in this strategy. The vehicle controller can determine the required torques of MG1 and MG2 based on the real-time vehicle speed and acceleration. This power distribution strategy can ensure optimal system efficiency and it satisfies the dynamic requirements of vehicles.
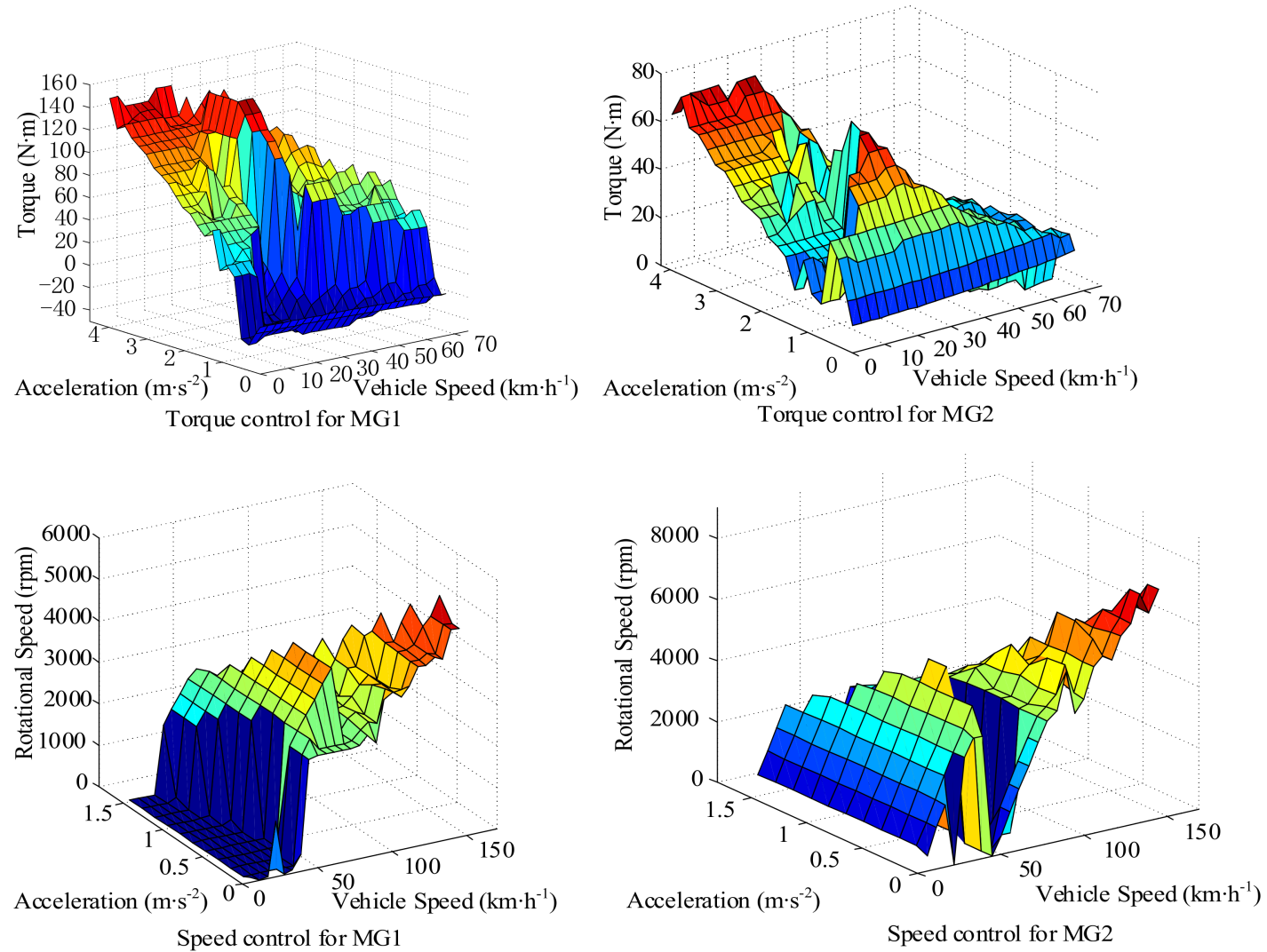

Figure 5. Power distribution strategy for main motor (MG1) and auxiliary motor (MG2).

\subsection{Mode-Switch Control Strategy}

This section presents the mode-switch control strategy, which not only has no power interruption during mode switching, but also guarantees smooth transition. The switching process between M1 and M4 is relatively complicated and representative because it includes the switching processes of other modes. Therefore, the switching process between M1 and M4 is investigated and presented in Table 4 .

Referring to Table 1 and Figure 1, the switching process presented in Table 4 enables the vehicle to switch between M1 and M4. During mode switching, the speed and torque of MG1 and MG2 are adjusted to meet the dynamic requirement of the vehicle without power interruption, which benefit 
from the introduction of the speed coupling mode and the torque coupling mode. However, the speed and torque of these motors need to increase or decrease in limited time in coupling modes during switching, which can be detrimental to the driving comfort.

Table 4. Switching process between single motor 1 drive (M1) and M4.

\begin{tabular}{|c|c|c|}
\hline Switch & Step & Process \\
\hline \multirow{3}{*}{ M1-to-M4 } & M1 to M3: & $\begin{array}{l}\text { (1) Start MG2 and regulate its speed to } \omega_{r} \cdot i_{0} \cdot i_{2} \cdot(k+1) \text { at no load; } \\
\text { (2) Engage clutch C3; Then, the torque of MG1 and MG2 can be adjusted. }\end{array}$ \\
\hline & M3 to M2: & $\begin{array}{l}\text { (1) Reduce the output torque of MG1 to zero and increase the torque of } \\
\text { MG2 to } T_{r} / i_{0} / i_{2} /(k+1) \text {; } \\
\text { (2) Disengage clutch C2 and decrease the speed of MG1 to zero. }\end{array}$ \\
\hline & M2 to M4: & $\begin{array}{l}\text { (1) Engage clutch } \mathrm{C} 1 \text {; } \\
\text { (2) Increase the torque of MG1 to } k T_{m 2} ; \\
\text { (3) Disengage brake B1; Then, the speed of MG1 and MG2 can be adjusted. }\end{array}$ \\
\hline \multirow{3}{*}{ M4-to-M1 } & M4 to M2: & $\begin{array}{l}\text { (1) Decrease the speed of MG1 to zero and increase the speed of MG2 to } \\
\omega_{r} \cdot i_{0} \cdot i_{2} \cdot(k+1) \text {; } \\
\text { (2) Engage brake B1; } \\
\text { (3) Decrease the torque of MG1 to zero; } \\
\text { (4) Disengage clutch } C 1 .\end{array}$ \\
\hline & M2 to M3: & $\begin{array}{l}\text { (1) Increase the speed of MG1 to } \omega_{r} \cdot i_{0} \cdot i_{1} \text {; } \\
\text { (2) Engage clutch C2; Then, the torque of MG1 and can be adjusted. }\end{array}$ \\
\hline & M3 to M1: & $\begin{array}{l}\text { (1) Decrease the torque of MG2 to zero and increase the torque of MG2 to } \\
T_{r} / i_{0} / i_{1} \text {; } \\
\text { (2) Disengage clutch } C 3 \text {; } \\
\text { (3) Decrease the speed of MG2 to zero. }\end{array}$ \\
\hline
\end{tabular}

This paper proposes the vehicle longitudinal jerk to estimate the vehicle ride performance. The jerk intensity $j$ is defined as the time derivative of acceleration $(\alpha)$, which must meet the national standard (the Chinese standard is $17 \mathrm{~m} / \mathrm{s}^{3}$ and the European Union standard is $10 \mathrm{~m} / \mathrm{s}^{3}$ ) [27]. The jerk intensity of M3 and M4 are modelled as Equations (8) and (9), respectively:

$$
\begin{gathered}
j=|\dot{\alpha}|=\left|\mathrm{r} \times \frac{\dot{T}_{m 2} \cdot(k+1) \cdot i_{2} \cdot \eta_{p} \cdot \eta_{2}+\dot{T}_{m 1} \cdot i_{1} \cdot \eta_{1}}{\left(\frac{I_{r}}{i_{0}{ }^{2}}+I_{m 2}(k+1)^{2} \cdot i_{2}^{2}+I_{m 1} \cdot i_{1}{ }^{2}\right) \times i_{0}}\right| \leq 10, \\
j=|\dot{\alpha}|=\left|\frac{I_{m 2} \cdot \dot{T}_{m 1} \cdot A \cdot \eta_{2} \cdot \eta_{0} \cdot k+I_{m 1} \cdot \dot{T}_{m 2} \cdot A \cdot \eta_{2} \cdot \eta_{0}}{I_{m 1} \cdot I_{m 2} \cdot A+I_{m 2} \cdot I_{r} \cdot k^{2}+I_{m 1} \cdot I_{r}} \cdot r\right| \leq 10,
\end{gathered}
$$

where $A=(k+1) \cdot i_{0} \cdot i_{2}$. Referring to Equations (8) and (9), the time derivative of the torque of MG1 and MG2 can be used to limit the jerk intensity during switch to satisfy the European Union standard.

\section{Simulation Results and Discussion}

\subsection{Simulation Model}

A simulation model for a PEV equipped with NDMPS was implemented in MATLAB/Simulink (R2014a), as shown in Figure 6. The simulation model includes a driving cycle module, driver module, required torque calculation module, vehicle driving control module, NDMPS module, and whole vehicle module. The control of the vehicle was implemented through real-time information transfer and cooperative operation of each module. 


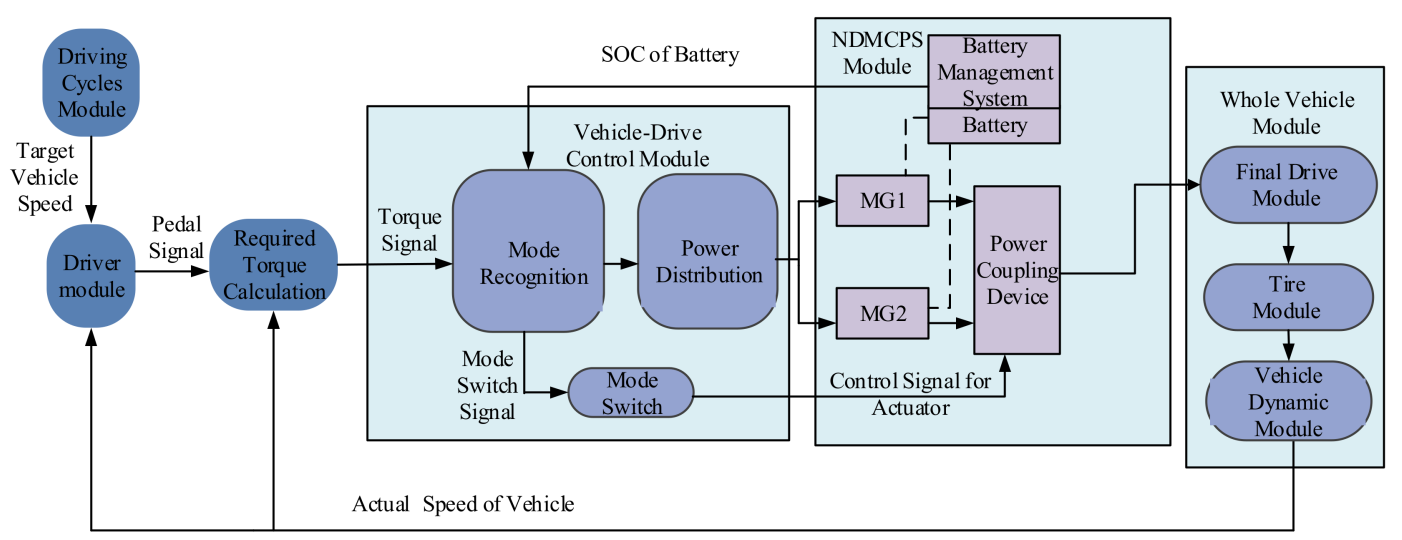

Figure 6. Simulation model for PEV equipped with NDMPS.

\subsection{Simulation and Verification of Mode Division Strategy.}

To verify the validity of the mode division strategy, model simulation was conducted in rapid acceleration conditions and the Worldwide Harmonised Light Vehicle Test Procedure (WLTP). The results are shown in Figure 7.
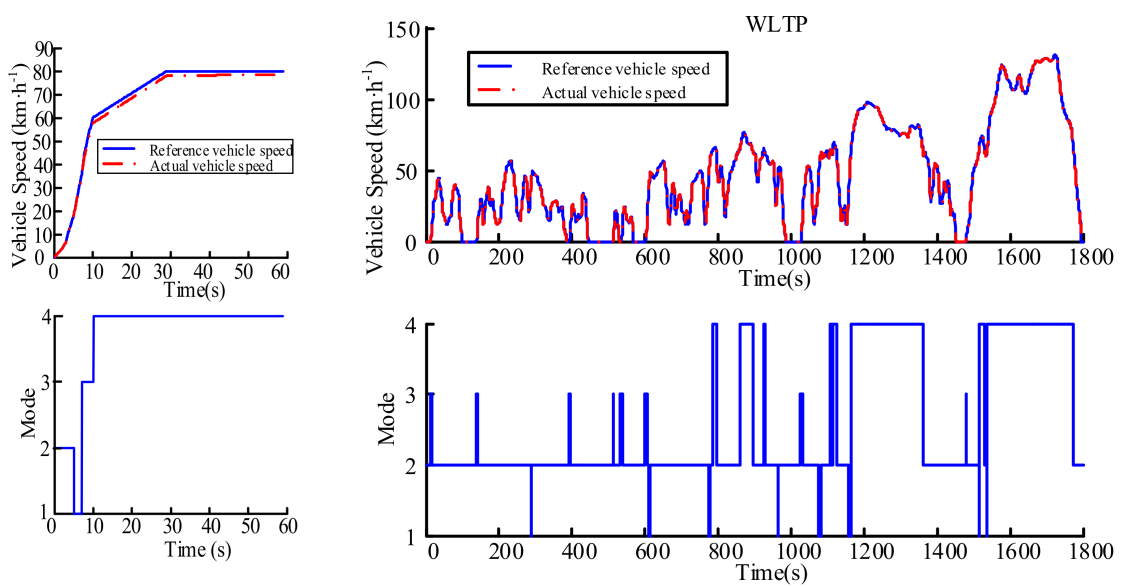

(a)

(b)

Figure 7. Simulation results for mode division under (a) rapid acceleration; (b) Worldwide Harmonised Light Vehicle Test Procedure (WLTP) drive cycle.

As shown in Figure 7, the actual vehicle speed tracks the reference speed very well, which indicates that the proposed control strategy can meet the requirement of vehicle dynamic performance. Figure $7 \mathrm{a}$ simulates the typical large acceleration process of vehicles, which included the switch of the four modes. The vehicle starts normally and accelerates rapidly to a certain velocity. Then, the acceleration pedal is released and the vehicle slowly accelerates to a relatively high speed. Accordingly, the simulation result shows that the vehicle starts with M2, and then switches to M1 and M3. Then, when the vehicle reaches a certain speed, it switches to M4. Figure $7 \mathrm{~b}$ shows that the mode distribution and division logic can select and switch modes reasonably in the WLTP drive cycle.

The simulation results in Figure 7 verify the validity of the mode division strategy that is proposed in this paper. Figure 7 indicates that the vehicle selects a reasonable driving mode to compensate the change in driving conditions. The vehicle tends to start at M2, and operates in M4 on reaching a certain speed. In the low-speed high-torque-requirement condition, for example, in the vehicle launch stage, M3 is often selected. These results are congruent with the original design objective of the NDMPS. 


\subsection{Simulation for Mode-Switch Control Strategy}

The MATLAB/Simulink simulation platform and the Sim Drivelines module were used to implement a multi-field physical model to verify the validity of the mode-switch control strategy. The switching process between M1 and M4 was simulated to verify the mode-switch control strategy and the results are shown in Figure 8 , where $n_{m 1}$ and $n_{m 2}$ are the speed of MG1 and MG2, respectively.

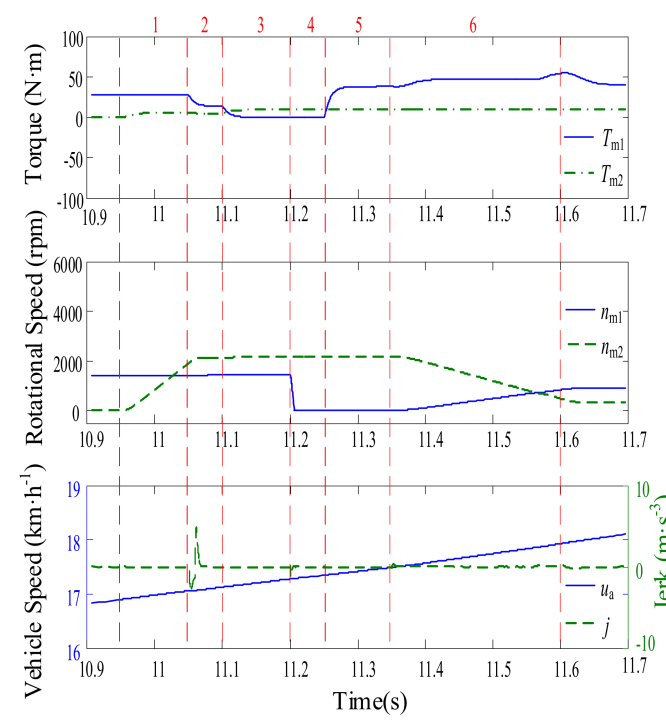

(a)

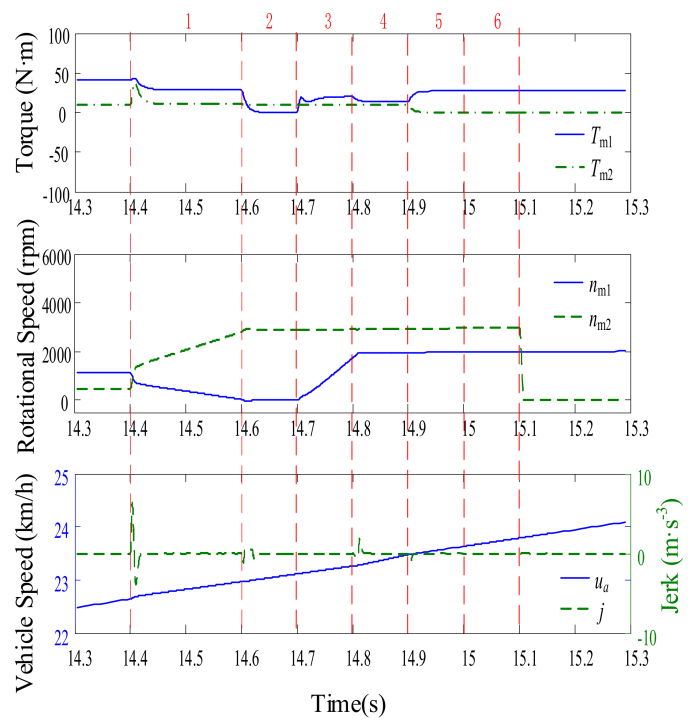

(b)

Figure 8. Simulation results of the switching process between M1 and M4: (a) M1-to-M4; and, (b) M4-to-M1.

As shown in Figure 8a, $0.65 \mathrm{~s}$ is expended in switching from M1 to M4. The system initially operates in M1 and receives a signal to carry out a mode switch at $10.95 \mathrm{~s}$. (1) From $10.95 \mathrm{~s}$ to $11.05 \mathrm{~s}$, MG2 is started and accelerated until its speed reaches $\omega_{r} \cdot i_{0} \cdot i_{2} \cdot(k+1) ;(2)$ From $11.05 \mathrm{~s}$ to $11.1 \mathrm{~s}, \mathrm{C} 3$ is engaged after the clutch driving disc and the driven disc are synchronized; (3) From $11.1 \mathrm{~s}$ to $11.2 \mathrm{~s}$, the torque of MG1 falls to zero and the torque of MG2 increases to $T_{r} / i_{0} / i_{2} /(k+1)$; (4) From $11.2 \mathrm{~s}$ to 11.25 s, C2 is disengaged and MG1 is stopped; (5) From 11.25 s to 11.35 s, C1 is engaged directly and the torque of MG1 increases to $k T_{m 2}$, equalising the torques on both sides of B1; (6) From $11.35 \mathrm{~s}$ to $11.6 \mathrm{~s}, \mathrm{~B} 1$ is released and the speed of MG1 and MG2 could be regulated within certain ranges. Finally, the system operates in M4.

In Figure 8a, the system controlled the actuators according to the established process during the switch from M1 to M4. There was no power interruption and the maximum jerk intensity during switching was $4.83 \mathrm{~m} / \mathrm{s}^{3}$, and this value is smaller than the limit value of $10 \mathrm{~m} / \mathrm{s}^{3}$.

As shown in Figure 8b, $0.7 \mathrm{~s}$ was expended in switching from M4 to M1. The system initially operates in M4 and received a signal to carry out a mode switch at $14.4 \mathrm{~s}$. (1) From $14.4 \mathrm{~s}$ to $14.6 \mathrm{~s}$, the speed of MG1 fall to zero, and the speed of MG2 increases to $\omega_{r} \cdot i_{0} \cdot i_{2} \cdot(k+1)$; (2) From $14.6 \mathrm{~s}$ to $14.7 \mathrm{~s}, \mathrm{~B} 1$ is engaged, the torque of MG1 decreased to zero, and C1 is disengaged; (3) From $14.7 \mathrm{~s}$ to $14.8 \mathrm{~s}$, the speed of MG1 increases to $\omega_{r} \cdot i_{0} \cdot i_{1}$, in preparation for the engagement of C2. (4) From $14.8 \mathrm{~s}$ to $14.9 \mathrm{~s}, \mathrm{C} 2$ is engaged after the driving plate and the driven plate were synchronized; (5) From $14.9 \mathrm{~s}$ to $15 \mathrm{~s}$, the torque of MG2 decreased to zero and the torque of MG1 increased to $T_{r} / i_{1} / i_{0} ;(6)$ From $15 \mathrm{~s}$ to $15.1 \mathrm{~s}, \mathrm{C} 3$ is disengaged, MG2 stopped driving, and its speed decreased to zero. Finally, the system operated in M1.

Figure $8 \mathrm{~b}$ shows that the system controlled the actuators according to the established process during the switch from M4 to M1. There is no power interruption and the maximum jerk during switching is $6.36 \mathrm{~m} / \mathrm{s}^{3}$, which is also smaller than the limit value of $10 \mathrm{~m} / \mathrm{s}^{3}$. 
Overall, the simulation results of the switching processes between M1 and M4 show that thFigur mode-switch strategy that is proposed in this paper is valid. This strategy controls the torque and the speed of motors during switching, which not only has no power interruption but also ensures operational smoothness.

\subsection{Comparative Simulation for PEVs}

To verify the superior performance of NDMPS, we compared it to a single-motor drive powertrain system (SMPS) that was designed in the study, having a two-speed transmission. The performance indicators of the SMPS were the same as those presented in Table 2 for NDMPS. Its basic parameters are shown in Table 5. The energy management strategy of the SMPS is also based on optimal system efficiency.

Table 5. Basic parameters of the single-motor drive powertrain system (SMPS).

\begin{tabular}{ccc}
\hline Item & Parameter & Value \\
\hline \multirow{3}{*}{ Motor } & Rated/peak power $(\mathrm{kW})$ & $35 / 67$ \\
& Rated/maximum speed $(\mathrm{rpm})$ & $2500 / 6300$ \\
& Rated/maximum torque $(\mathrm{N} \cdot \mathrm{m})$ & $124 / 253$ \\
\hline Final Drive & Ratio & 5.2 \\
\hline \multirow{2}{*}{ Transmission } & First gear ratio & 1.6 \\
& Second gear ratio & 1 \\
\hline
\end{tabular}

Figure 9 shows the dynamic performance of both the SMPS and NDMPS. As shown in Figure 9a, the SMPS and NDMPS have similar maximum torques under each vehicle speed. This similar external characteristic indicates that the parameters of the SMPS are well matched. Figure $9 \mathrm{~b}$ presents the acceleration time of both powertrain systems. It shows that NDMPS has a better acceleration performance than the SMPS during vehicle launch. The details of the dynamic performance of both systems are presented in Table 6.

Table 6. Comparison of vehicle dynamic performance.

\begin{tabular}{|c|c|c|c|}
\hline Item & SMPS & NDMPS & Increment $(\%)$ \\
\hline Maximum speed $(\mathrm{km} / \mathrm{h})$ & 160 & 160 & - \\
\hline $\begin{array}{l}\text { Acceleration time of } \\
0 \sim 50 \mathrm{~km} / \mathrm{h}(\mathrm{s})\end{array}$ & 4.85 & 4.6 & $5.43 \%$ \\
\hline $\begin{array}{c}\text { Stable speed at } 30 \% \\
\text { climbing grade }(\mathrm{km} / \mathrm{h})\end{array}$ & 43.7 & 43.7 & - \\
\hline
\end{tabular}

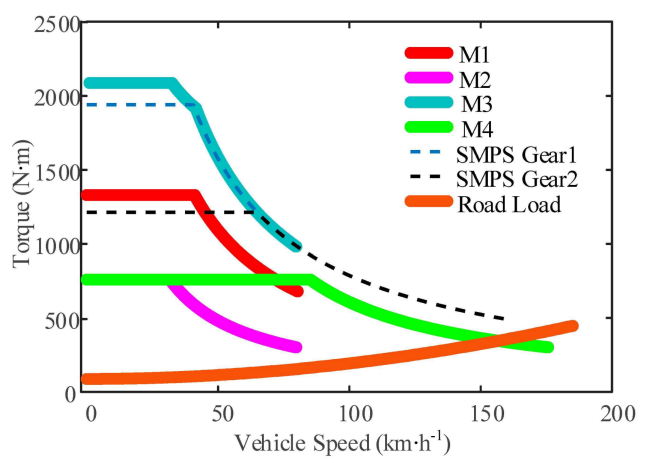

(a)

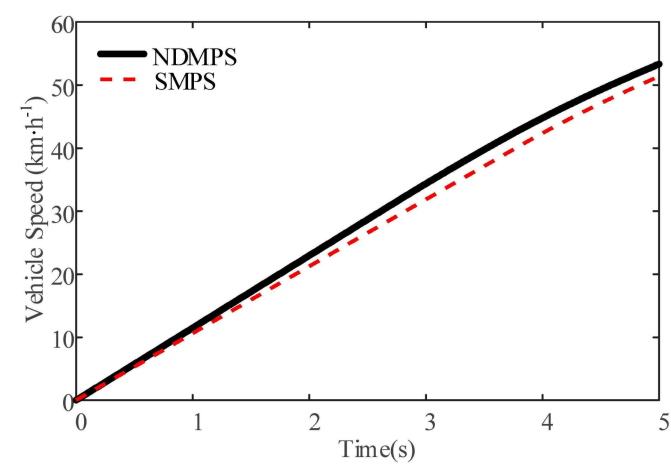

(b)

Figure 9. Dynamics simulation for the SMPS and NDMPS. (a) External characteristics of the driving system; (b) Acceleration time. 
The economic performance of a PEV, respectively, equipped with NDMPS and SMPS, was also analysed. The results are presented in Figure 10 and Table 7. In the mileage simulation, the initial value of $S O C$ of battery pack is 0.9 and the simulation stops when the SOC drops to 0.3 .
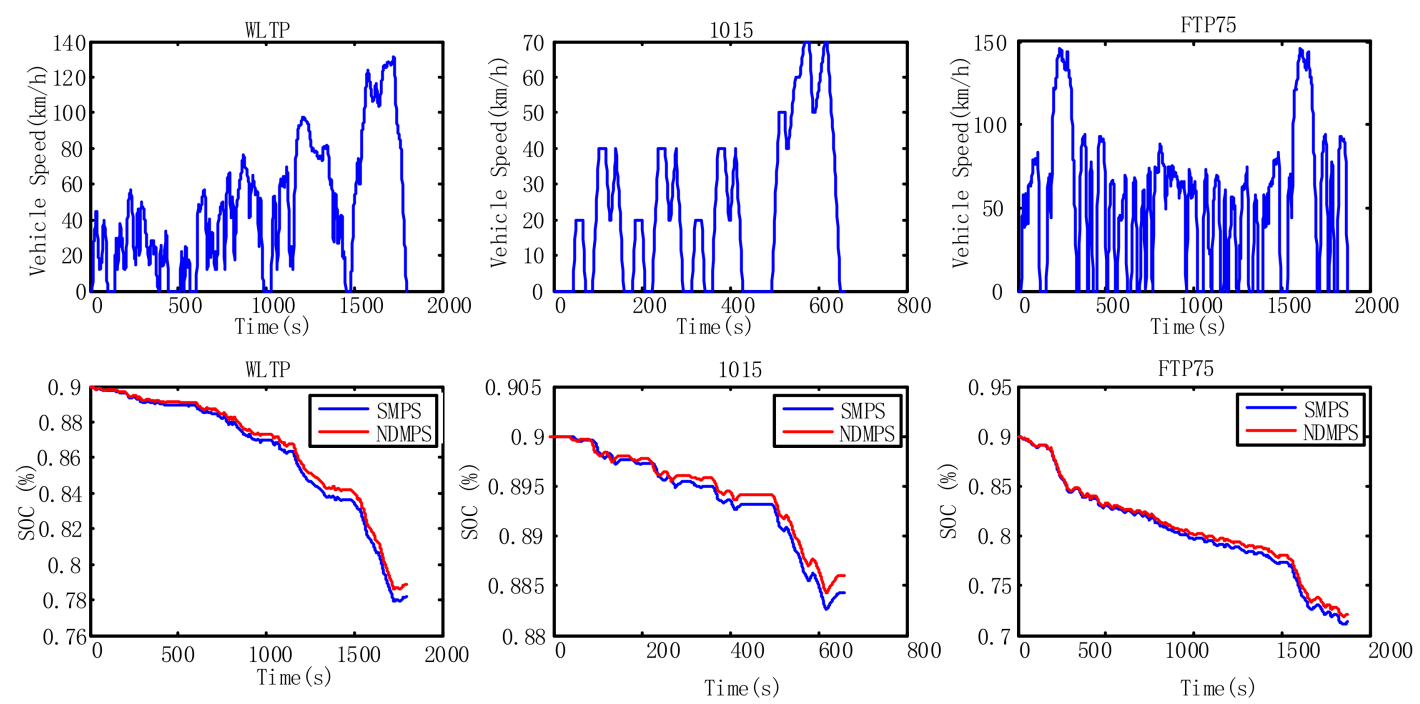

Figure 10. Simulation results of state of charge $(S O C)$ under different driving cycles.

Table 7. Comparison of vehicle economic performance.

\begin{tabular}{cccc}
\hline Driving Condition & SMPS (km) & NDMPS (km) & Increment (\%) \\
\hline Mileage of 60 & 157.5 & 163.3 & $3.55 \%$ \\
km/h cruising & 114.5 & 125.2 & $8.56 \%$ \\
WLTP & 154.9 & 174.2 & $11.1 \%$ \\
1015 & 88.5 & 93.5 & $5.35 \%$ \\
FTP75 & & & \\
\hline
\end{tabular}

As shown in Figure 10 and Table 7, NDMPS has better economic performance than that of SMPS. In Figure 10, the battery SOC of NDMPS tends to drop slower than the SMPS. This is because the NDMPS have larger high system efficiency region than SMPS. Therefore, for giving vehicle speed and acceleration, the required power of battery for NDMPS is prone to be smaller. The mileages in the cruising condition and the different driving cycles (Table 7) show various degrees of improvement. Specifically, the mileage at $60 \mathrm{~km} / \mathrm{h}$ cruising for NDMPS is $3.35 \%$ higher than that of SMPS and the maximum increment percentage for mileage among WLTP, 1015, and FTP75 (the EPA Federal Test Procedure) is $11.1 \%$. This also indicates the potential energy-saving capability of NDMPS.

\section{Conclusions}

In this study, a novel dual-motor coupling-propulsion powertrain system (NDMPS) was proposed and simulated. The proposed NDMPS can realize four driving modes: single motor 1 drive, single motor 2 drive, two motor torque coupling drive, and two motor speed coupling drive. The operational characteristics of each driving mode of NDMPS were subsequently analysed, and, based on the optimal system efficiency rule, the working point of each mode was identified. Mode division and motor power distribution strategies were designed. A mode-switch control strategy that reduces jerk during mode switching by controlling the torque variation rate of the motors, thereby ensuring driving comfort during mode switching, was also proposed. The vehicle model simulation results verify the reliability and the validity of the mode division strategy and smoothness during mode switching. Further, a comparison of the simulation results of a single-motor drive PEV to that of the proposed dual-motor 
drive PEV shows that the acceleration time for $0-50 \mathrm{~km} / \mathrm{h}$ of the dual-motor PEV is $5.43 \%$ lower than that of the single-motor PEV. In addition, the energy efficiency of the dual-motor PEV in the constant-speed cruising condition, WLTP, 1015, and FTP75 drive cycle showed improvements of 3.55\%, $8.56 \%, 11.1 \%$, and $5.35 \%$, respectively. These results demonstrate the superior dynamic performances and energy-saving capability of the proposed NDMPS.

Acknowledgments: This work was supported by the Chongqing Natural Science Foundation [grant number cstc2015jcyjA60005] and the National Key Research and Development Program of China [grant number 2016YFB0101402). The projects offered all the costs of this series researches. The authors appreciate for their supports on these researches.

Author Contributions: Jianjun Hu wrote the first draft of the manuscript, developed the novel powertrain system and the drive control strategy, and analysed the data. Lingling Zheng defined the primary simulation framework and carried out the simulation. Meixia Jia set up the single-motor drive powertrain model and performed its related simulation. Yi Zhang and Tao Pang provided insights and additional ideas on presentation. All authors revised and approved the manuscript.

Conflicts of Interest: The authors declare no conflict of interest.

\section{References}

1. Chu, S.; Majumdar, A. Opportunities and challenges for a sustainable energy future. Nature 2012, 488, 294-303. [CrossRef] [PubMed]

2. Rahbari-Asr, N.; Chow, M.Y. Cooperative distributed demand management for community charging of PHEV/PEVs based on KKT conditions and consensus networks. IEEE Trans. Ind. Inform. 2014, 10, 1907-1916. [CrossRef]

3. Crolla, D.A.; Cao, D. The impact of hybrid and electric powertrains on vehicle dynamics, control systems and energy regeneration. Veh. Syst. Dyn. 2012, 50, 95-109. [CrossRef]

4. Miller, M.; Holmes, A.; Conlon, B.; Savagian, P. The GM “Voltec"4ET50 multi-mode electric transaxle. SAE Int. J. Engines 2011, 4, 1102-1114. [CrossRef]

5. Wu, X.; Han, S.; Sun, H.; Han, G. Efficiency analysis of planetary coupling drive system with dual motors on electric bus. In Proceedings of the 2015 IEEE International Conference on Mechatronics and Automation (ICMA), Beijing, China, 2-5 August 2015; pp. 2547-2552.

6. Zhang, S.; Zhang, C.; Han, G.; Wang, Q. Optimal control strategy design based on dynamic programming for a dual-motor coupling-propulsion system. Sci. World J. 2014. [CrossRef] [PubMed]

7. Kim, J.; Kim, T.; Min, B.; Hwang, S.; Kim, H. Mode Control Strategy for a Two-Mode Hybrid Electric Vehicle Using Electrically Variable Transmission (EVT) and Fixed-Gear Mode. IEEE Trans. Veh. Technol. 2011, 60, 793-803. [CrossRef]

8. Musardo, C.; Rizzoni, G.; Guezennec, Y.; Staccia, B. A-ECMS: An adaptive algorithm for hybrid electric vehicle energy management. Eur. J. Control 2005, 11, 509-524. [CrossRef]

9. Xi, L.; Zhang, X.; Sun, C.; Wang, Z.; Hou, X.; Zhang, J. Intelligent Energy Management Control for Extended Range Electric Vehicles Based on Dynamic Programming and Neural Network. Energies 2017, 10, 1871. [CrossRef]

10. Zou, Y.; Sun, F.; Hu, X. Combined Optimal Sizing and Control for a Hybrid Tracked Vehicle. Energies 2012, 5, 4697-4710. [CrossRef]

11. Un-Noor, F.; Padmanaban, S.; Mihet-Popa, L.; Mollah, M.N.; Hossain, E. A comprehensive study of key electric vehicle (EV) components, technologies, challenges, impacts, and future direction of development. Energies 2017, 10, 1217. [CrossRef]

12. Tie, S.F.; Tan, C.W. A review of energy sources and energy management system in electric vehicles. Renew. Sustain. Energy Rev. 2013, 20, 82-102. [CrossRef]

13. Guan, J.C.; Chen, B.C. Adaptive Power Management Strategy for a Four-Mode Hybrid Electric Vehicle. Energy Procedia 2017, 105, 2403-2408. [CrossRef]

14. Sciarretta, A.; Guzzella, L. Control of hybrid electric vehicles. IEEE Control Syst. 2007, 27, 60-70. [CrossRef]

15. Thibault, L.; Grondin, S.O.; Quérel, C.; Corde, G. Energy Management Strategy and Optimal Hybridization Level for a Diesel HEV; SAE World Congress: Detroit, MI, USA, 2012; pp. 260-271.

16. Zhang, C.; Zhang, S.; Han, G.; Liu, H. Power Management Comparison for a Dual-Motor-Propulsion System Used in a Battery Electric Bus. IEEE Trans. Ind. Electron. 2017, 64, 3873-3882. [CrossRef] 
17. Da, W.; Bo, W. Research on driving force optimal distribution and fuzzy decision control system for a dual-motor electric vehicle. In Proceedings of the 2015 34th Chinese Control Conference (CCC), Hangzhou, China, 28-30 July 2015; pp. 8146-8153.

18. Li, L.; Yang, C.; Zhang, Y.; Zhang, L.; Song, J. Correctional DP-Based Energy Management Strategy of Plug-In Hybrid Electric Bus for City-Bus Route. IEEE Trans. Veh. Technol. 2015, 64, 2792-2803. [CrossRef]

19. Moura, S.J.; Fathy, H.K.; Callaway, D.S.; Stein, J.L. A Stochastic Optimal Control Approach for Power Management in Plug-In Hybrid Electric Vehicles. IEEE Trans. Control Syst. Trans. 2011, 19, 545-555. [CrossRef]

20. Dizqah, A.M.; Lenzo, B.; Sorniotti, A.; Gruber, P.; Fallah, S.; De Smet, J. A fast and parametric torque distribution strategy for four-wheel-drive energy-efficient electric vehicles. IEEE Trans. Ind. Electron. 2016, 63, 4367-4376. [CrossRef]

21. He, H.; Liu, Z.; Zhu, L. Dynamic Coordinated Shifting Control of Automated Mechanical Transmissions without a Clutch in a Plug-In Hybrid Electric Vehicle. Energies 2012, 5, 3094-3109. [CrossRef]

22. Ma, Y.; Xiang, C.; Huang, K.; Liu, H.; Li, Y. Modelling and mode switch stability criterion research of power-split hybrid transmission system. In Proceedings of the International Conference on Modelling, Identification and Control, Melbourne, Australia, 3-5 December 2015; pp. 225-230.

23. Sim, K.; Oh, S.-M.; Namkoong, C.; Lee, J.-S.; Han, K.-S.; Hwang, S.-H. Control strategy for clutch engagement during mode change of plug-in hybrid electric vehicle. Int. J. Automot. Technol. 2017, 18, 901-909. [CrossRef]

24. Wang, J.; Wang, Q.; Wang, P.; Li, L. Mode Transition Dynamic Control for Dual-Motor Hybrid Driving System. In Proceedings of the SAE/KSAE 2013 International Powertrains, Fuels Lubricants Meeting, Seoul, Korean, 14 October 2013.

25. Hu, M.; Zeng, J.; Xu, S.; Fu, C. Efficiency Study of a Dual-Motor Coupling EV Powertrain. IEEE Trans. Veh. Technol. 2015, 64, 2252-2260. [CrossRef]

26. Guo, L.; Gao, B.; Gao, Y.; Chen, H. Optimal energy management for HEVs in eco-driving applications using bi-level MPC. IEEE Trans. Intell. Transp. Syst. 2017, 18, 2153-2162. [CrossRef]

27. Zhao, Z.; Lei, D.; Chen, J.; Li, H. Optimal control of mode transition for four-wheel-drive hybrid electric vehicle with dry dual-clutch transmission. Mech. Syst. Signal Process 2018, 105, 68-89. [CrossRef]

(C) 2018 by the authors. Licensee MDPI, Basel, Switzerland. This article is an open access article distributed under the terms and conditions of the Creative Commons Attribution (CC BY) license (http:/ / creativecommons.org/licenses/by/4.0/). 